POS PROCEEDINGS

\title{
Status of Composite Higgs
}

\section{Aleksandr Azatov*防}

SISSA/ISAS and INFN, I-34136 Trieste, Italy

E-mail: aazatov@sissa.it

In these proceedings I will briefly describe the Composite Higgs constructions and then discuss their current experimental status.

The European Physical Society Conference on High Energy Physics

5-12 July, 2017

Venice

* Speaker.

${ }^{\dagger}$ I would like to thank the conveners of the "Higgs and New Physics " session for the invitation. 


\section{Introduction}

The discovery of the Higgs boson poses in front of the particle physics community a crucial question about its nature? Whether it is indeed the first elementary scalar to be found in nature or a composite boson of a new strong dynamics. The idea of a Composite Higgs $(\mathrm{CH})$ appeared in the late 80s by Georgi and Kaplan[1], and in this class of models the Higgs boson is a pseudo NambuGoldstone boson(pNGB) appearing after the confinement of a new strongly interacting sector. This construction helps to avoid the Higgs mass hierarchy problem as well as explain why the new scalar is so much lighter than the rest of the composite resonances and has properties similar to the elementary particle (for the detailed review of the phenomenological and theoretical properties of the $\mathrm{CH}$ see for example $[2,3])$.

\section{Minimal construction and bounds}

The backbone of the $\mathrm{CH}$ models lies on the assumption of a new confining dynamics invariant under the global group $G$ broken spontaneously during the confinement to its subgroup $H$. The resulting Goldstone bosons appear as a multiplet of the coset $G / H$, and the Standard Model (SM) Higgs boson is supposed to be one of them. One can think about the $\mathrm{CH}$ models as a scaled up version of the QCD where the Higgs boson is an analogue of the pion fields . The further gauging of the subgroup $H$ (or its part) as well as the Higgs boson Yukawa interactions with the SM fields lead to the explicit breaking of the Goldstone symmetry and appearance of the Higgs potential.

The current constraints on the electroweak precision observables restrict the coset constructions to contain custodial $S U(2)_{L} \times S U(2)_{R}$ symmetry as a part of the unbroken subgroup. Then the minimal model where electroweak Higgs doublet can be embedded is based on $S O(5) / S O(4)$ symmetry breaking [4]. CCWZ description of the nonlinearly realized symmetries [5, 6] allows us to derive the interactions between the $\mathrm{CH}$ and the SM gauge bosons:

$$
\begin{gathered}
\frac{f^{2}}{4} \operatorname{Tr}\left(D_{\mu} U\right)^{\dagger} D^{\mu} U=\frac{1}{2}\left(\partial_{\mu} h\right)^{2}+\frac{f^{2}}{4}\left(g^{2} W_{\mu}^{+} W_{\mu}^{-}+\frac{g^{2}}{2 c_{W}^{2}} Z_{\mu}^{2}\right) \sin ^{2}\left(\theta+\frac{h}{f}\right) \\
U(\Pi)=e^{i \Pi / f}, \Pi=\Pi^{\hat{a}} T^{\hat{a}},
\end{gathered}
$$

where the $\theta$ is the misalignment angle between the unbroken subgroup and the one defined by the SM gauging. The modifications of the Higgs couplings to the gauged fields compared to the SM values are equal to:

$$
\frac{g_{h v v}^{\text {Composite Higgs }}}{g_{h v v}^{S M}}=\cos (\theta)=\sqrt{1-\frac{v_{S M}^{2}}{f^{2}}}=\sqrt{1-\xi}, \quad \xi \equiv \frac{v_{S M^{2}}}{f^{2}} .
$$

Current LHC Higgs couplings measurements constrain this modification to be

$$
\frac{v_{S M^{2}}}{f^{2}}<0.1 @ 95 \% \Rightarrow f \gtrsim 800 \mathrm{GeV},
$$

which leads to the lower bound on the scale of the Higgs compositeness. Notice that the same parameter $\xi$ controls the tuning in the scale of the EWSB, since from dimensional analysis the natural value of the Higgs vev should be given by the scale of composite symmetry breaking. In spite of the big success in the measurements of the Higgs couplings at LHC, the strongest 
constraints on $\xi$ are still coming from the LEP experiment. Namely EW precision parameters are getting new IR contribution due to the modifications of the Higgs couplings:

$$
\begin{gathered}
S_{I R}=\frac{1}{12 \pi}\left[\log \frac{m_{h}^{2}}{m_{Z}^{2}}+\xi \log \frac{\Lambda^{2}}{m_{h}^{2}}\right], \Lambda \sim \frac{4 \pi v_{S M}}{\sqrt{\xi}} \\
T_{I R}=\frac{-3}{16 \pi \cos ^{2} \theta_{W}}\left[\log \frac{m_{h}^{2}}{m_{Z}^{2}}+\xi \log \frac{\Lambda^{2}}{m_{h}^{2}}\right] .
\end{gathered}
$$

These effects lead to the very strong constraints on the scale of compositeness

$$
\xi<0.02 @ 95 \%, f \gtrsim 1.2 \mathrm{TeV},
$$

requiring at least percent level of tuning. Even more the masses of the new resonances $m_{*} \sim g_{*} f$ are getting close to the limits of the LHC discovery possibilities $m_{*} \sim g_{*} f$ unless we are dealing with "weakly" coupled versions of the strong dynamics. At the same time I would like to stress that this bound is less robust than Eq. 2.3, since IR loop contribution to the electroweak precision parameters can be cancelled by the new UV contributions at a price of additional tuning. Indeed, loops composite resonances can lead to the large contribution to the $\mathrm{S}$ parameter [7] and the $\mathrm{T}$ parameter can receive large modifications due to the mixing between SM and the strong sector [8] (see right of the Fig.2).

\subsection{Vector resonances}

Let us look now at the new particle content of the models. By analogy with QCD we expect new composite resonances with the masses $\sim g_{*} f$, and these new resonances should appear as the multiplets of the unbroken subgroup i.e. $S U(2)_{L} \times S U(2)_{R}$. The simplest options are EW triplets and singlets. These fields will couple to the Standard model electroweak currents (similarly to the $\rho$ photon mixing in $\mathrm{QCD}$ ) with the strength:

$$
\alpha J_{\mu}^{a} \rho_{\mu}^{a}, \quad \alpha \sim \frac{g^{2}}{g_{*}}
$$

where the $g, g_{*}$ are the SM and the strong sector couplings respectively. At the same time the couplings of the new fields to the longitudinal components of the SM gauge bosons will be enhanced due to the composite origin of the Higgs boson

$$
\beta \varepsilon^{a b c} \rho_{\mu}^{a} W_{v}^{b} \partial_{v} W_{\mu}^{c}, \quad \beta \sim \frac{m_{*}^{2} E}{g_{*} f^{2}} .
$$

This simple qualitative analysis shows that the composite vector bosons should be dominantly produced in the Drell-Yan process with the suppressed coupling to light generation quarks compared to the SM Eq. 2.6 and then decay mostly into the longitudinal components of the SM vector bosons. This process is analysed by both ATLAS and CMS collaborations and $[9,10]$ and the current bounds on the new vector masses is around $m_{\rho} \gtrsim 3 \mathrm{TeV}$, however the bounds become looser for the values $g_{\rho} \gtrsim 3$ due to the suppressed production rate and increase of the width of the resonance. Competitive bounds can be obtained from the EW precision tests. Tree level diagrams with the virtual $\rho$ boson exchange generate corrections to the $S$ parameter of the order $\Delta S \sim \frac{4 \pi v^{2}}{M_{*}^{2}}$ requiring $m_{\rho} \gtrsim 2 \mathrm{TeV}$ ( for the one loop effects see $[11,12,13]$ ). 


\section{SM fermions in Composite Higgs models}

So far we have been focusing mostly on the SM vector bosons and their interactions with the composite sector, but what about the SM fermions? How these fields can be incorporated in the $\mathrm{CH}$ picture? One of the most popular possibilities helping to reduce dangerous flavor violation effects from the composite sector arises within the partial compositeness paradigm [14]. The basic construction is the following: SM model fermions are considered to belong to the "elementary" sector and these fields further mix only linearly with the operators of the strong sector:

$$
\Delta \mathscr{L}=\frac{\lambda_{t_{L}}}{\Lambda_{u \nu}^{d_{L}-5 / 2}} \bar{q}_{L} O^{L}+\frac{\lambda_{t_{R}}}{\Lambda_{U V}^{d_{R}-5 / 2}} t_{R} O_{R}
$$

The confinement of the strongly coupled sector leads to the formation of the bound fermions states which mix linearly with the SM fermions. This mixing between elementary and composite fermions generates the Yukawa interactions between SM fermions. If the interactions in Eq.3.1 are irrelevant, exponentially different values of the mixing between the SM and composite sector can be easily achieved by small variations of the corresponding anomalous dimensions

$$
\sin _{L, R} \sim\left(\frac{\Lambda_{I R}}{\Lambda_{u v}}\right)^{d_{L, R}-5} m \sim\left(\frac{\Lambda_{I R}}{\Lambda_{u v}}\right)^{d_{L}+d_{R}-5} .
$$

This effect can be used to explain the hierarchies in the fermion mass sector and simultaneously suppress the flavor violation [15]. In this case the strongest bounds come from the diagrams with the composite gluon exchange $[16,17]$ leading to the $M_{\text {comp. gluon }} \gtrsim 15 \mathrm{TeV}$, which makes the most of the composite resonances hardly (if at all) observable at LHC [18, 19]. Of course the masses of the composite sector can be made lighter turning to some partial cancellations/tuning in the flavor sector or implementing flavor symmetries [20]. Having in mind this tension from the flavor observables let us nevertheless look at the main collider signatures of the partial compositenes.

\section{Bounds on top partners}

SM gauge and Yukawa interactions break the Goldstone symmetry of the Higgs boson explicitly, leading to the generation of the Higgs potential. The largest symmetry breaking spurion is the SM top Yukawa coupling, so that the potential of Higgs is pretty much controlled by the interactions between the top quark and the strongly coupled sector. This leads to the indirect relation between the Higgs mass and the masses of the composite top partners [21, 22, 23, 24]:

$$
m_{H}^{2} \sim \frac{m_{t}^{2} M_{*}^{2}}{16 \pi^{2} f^{2}}
$$

clearly indicating the preference for the light top partners. The formula 4.1 is derived for the model where the top quark is mixed with 5 representation of $\mathrm{SO}(5)$, however similar results will apply for the other representations as well[25]. The new fields (top partners) will appear in the multiplets of $S U(2)_{L} \times S U(2)_{R}$ so that generically there will be new coloured fermions with exotic charges $5 / 3^{1}$. These fields will further decay into SM top quark and $\mathrm{W}$ boson leading to the same sign lepton in the final state. The main production channel will be either QCD pair production or singly in association with top or bottom quarks (see Fig.1) [27, 28, 29, 30, 31, 32]. The current bounds 

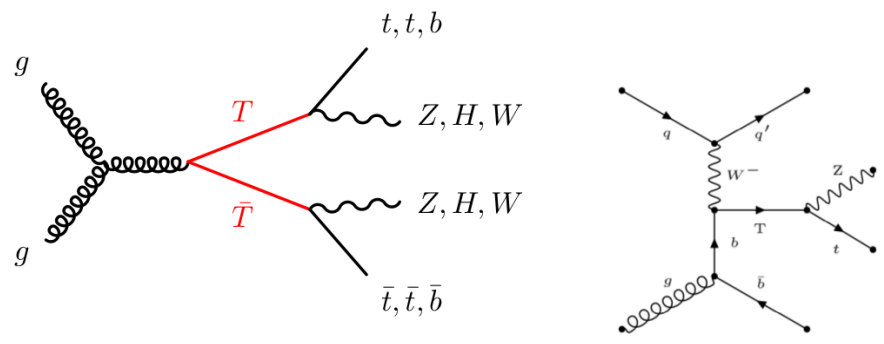

Figure 1: Feynman diagrams contributing to the pair and single production of top parnters.
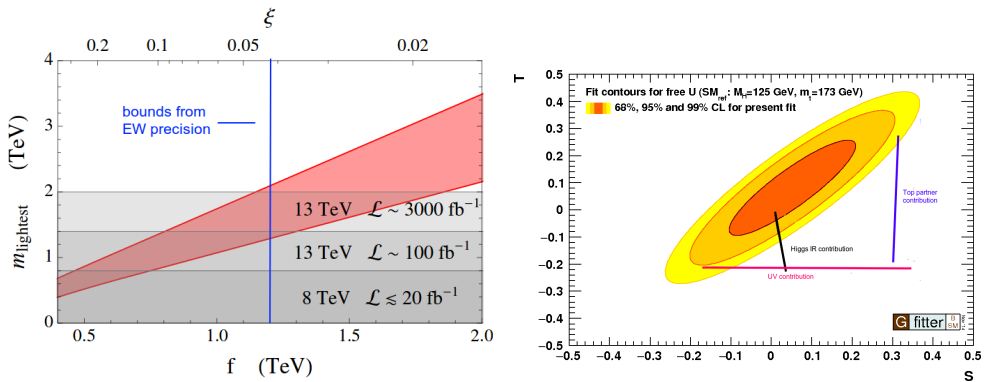

Figure 2: Left- prospects for the the LHC constraints at high luminosity. The red ban indicates the values of the top partners allowed by the current measurements of the Higgs mass (the figure is taken from [30]). Right- Current constraints on the EW precision $S$, and $T$ parameters [33], black line indicates the the contributions from the loops of the Higgs boson with the modified couplings Eq. 2.4 . Red and blue lines indicate UV contribution to the S parameters and the contribution to the T parameter from the loops of top partners.

range in $M_{*} \gtrsim 1-1.3 \mathrm{TeV}$. So far the bounds from the direct searches are weaker than the ones from the electroweak precision tests see Fig.2, however LHC at high luminosity will be able to put a very competetive constraints.

\section{Conclusion}

In conclusion I would like to say that the composite Higgs models provide an interesting solution to the Higgs mass hierarchy problem. However both direct and indirect searches tell that this scenario can be compatible with reality if only there is some extra tuning in the Higgs boson potential. The LHC reach in the searches of the new particles is still far from saturation and the new resonances could be just sitting behind the corner, so let us wait for the new data!

\section{References}

[1] D. B. Kaplan and H. Georgi, Phys. Lett. 136B, 183 (1984). doi:10.1016/0370-2693(84)91177-8

\footnotetext{
${ }^{1}$ Inspite of the large multiplicities of the new composite states charged under QCD the couplings of the Higgs boson to the gluons and photons are affected only mildly [26] due to the Goldstone symmetry.
} 
[2] G. Panico and A. Wulzer, Lect. Notes Phys. 913, pp.1 (2016) doi:10.1007/978-3-319-22617-0 [arXiv:1506.01961 [hep-ph]].

[3] R. Contino, arXiv:1005.4269 [hep-ph].

[4] K. Agashe, R. Contino and A. Pomarol, Nucl. Phys. B 719, 165 (2005) doi:10.1016/j.nuclphysb.2005.04.035 [hep-ph/0412089].

[5] S. R. Coleman, J. Wess and B. Zumino, Phys. Rev. 177, 2239 (1969). doi:10.1103/PhysRev.177.2239

[6] C. G. Callan, Jr., S. R. Coleman, J. Wess and B. Zumino, Phys. Rev. 177, 2247 (1969). doi:10.1103/PhysRev.177.2247

[7] A. Azatov, R. Contino, A. Di Iura and J. Galloway, Phys. Rev. D 88, no. 7, 075019 (2013) doi:10.1103/PhysRevD.88.075019 [arXiv:1308.2676 [hep-ph]].

[8] C. Grojean, O. Matsedonskyi and G. Panico, JHEP 1310, 160 (2013) doi:10.1007/JHEP10(2013)160 [arXiv:1306.4655 [hep-ph]].

[9] A. M. Sirunyan et al. [CMS Collaboration], arXiv:1708.05379 [hep-ex].

[10] M. Aaboud et al. [ATLAS Collaboration], arXiv:1708.09638 [hep-ex].

[11] A. Orgogozo and S. Rychkov, JHEP 1203, 046 (2012) doi:10.1007/JHEP03(2012)046 [arXiv:1111.3534 [hep-ph]].

[12] A. Pich, I. Rosell and J. J. Sanz-Cillero, JHEP 1401, 157 (2014) doi:10.1007/JHEP01(2014)157 [arXiv:1310.3121 [hep-ph]].

[13] R. Contino and M. Salvarezza, Phys. Rev. D 92, no. 11, 115010 (2015) doi:10.1103/PhysRevD.92.115010 [arXiv:1511.00592 [hep-ph]].

[14] D. B. Kaplan, Nucl. Phys. B 365, 259 (1991). doi:10.1016/S0550-3213(05)80021-5

[15] K. Agashe, G. Perez and A. Soni, Phys. Rev. D 71, 016002 (2005) doi:10.1103/PhysRevD.71.016002 [hep-ph/0408134].

[16] C. Csaki, A. Falkowski and A. Weiler, JHEP 0809, 008 (2008) doi:10.1088/1126-6708/2008/09/008 [arXiv:0804.1954 [hep-ph]].

[17] K. Agashe, A. Azatov and L. Zhu, Phys. Rev. D 79, 056006 (2009) doi:10.1103/PhysRevD.79.056006 [arXiv:0810.1016 [hep-ph]].

[18] A. Azatov, D. Chowdhury, D. Ghosh and T. S. Ray, JHEP 1508, 140 (2015) doi:10.1007/JHEP08(2015)140 [arXiv:1505.01506 [hep-ph]].

[19] J. P. Araque, N. F. Castro and J. Santiago, JHEP 1511, 120 (2015) doi:10.1007/JHEP11(2015)120 [arXiv:1507.05628 [hep-ph]].

[20] M. Redi and A. Weiler, JHEP 1111, 108 (2011) doi:10.1007/JHEP11(2011)108 [arXiv:1106.6357 [hep-ph]].

[21] D. Marzocca, M. Serone and J. Shu, JHEP 1208, 013 (2012) doi:10.1007/JHEP08(2012)013 [arXiv:1205.0770 [hep-ph]].

[22] O. Matsedonskyi, G. Panico and A. Wulzer, JHEP 1301, 164 (2013) doi:10.1007/JHEP01(2013)164 [arXiv:1204.6333 [hep-ph]].

[23] M. Redi and A. Tesi, JHEP 1210, 166 (2012) doi:10.1007/JHEP10(2012)166 [arXiv:1205.0232 [hep-ph]]. 
[24] A. Pomarol and F. Riva, JHEP 1208, 135 (2012) doi:10.1007/JHEP08(2012)135 [arXiv:1205.6434 [hep-ph]].

[25] G. Panico, M. Redi, A. Tesi and A. Wulzer, JHEP 1303, 051 (2013) doi:10.1007/JHEP03(2013)051 [arXiv:1210.7114 [hep-ph]].

[26] A. Azatov and J. Galloway, Phys. Rev. D 85, 055013 (2012) doi:10.1103/PhysRevD.85.055013 [arXiv:1110.5646 [hep-ph]].

[27] A. De Simone, O. Matsedonskyi, R. Rattazzi and A. Wulzer, JHEP 1304, 004 (2013) doi:10.1007/JHEP04(2013)004 [arXiv:1211.5663 [hep-ph]].

[28] A. Azatov, O. Bondu, A. Falkowski, M. Felcini, S. Gascon-Shotkin, D. K. Ghosh, G. Moreau and S. Sekmen, Phys. Rev. D 85, 115022 (2012) doi:10.1103/PhysRevD.85.115022 [arXiv:1204.0455 [hep-ph]].

[29] A. Azatov, M. Salvarezza, M. Son and M. Spannowsky, Phys. Rev. D 89, no. 7, 075001 (2014) doi:10.1103/PhysRevD.89.075001 [arXiv:1308.6601 [hep-ph]].

[30] O. Matsedonskyi, G. Panico and A. Wulzer, JHEP 1604, 003 (2016) doi:10.1007/JHEP04(2016)003 [arXiv:1512.04356 [hep-ph]].

[31] M. Aaboud et al. [ATLAS Collaboration], JHEP 1708, 052 (2017) doi:10.1007/JHEP08(2017)052 [arXiv:1705.10751 [hep-ex]].

[32] CMS Collaboration [CMS Collaboration], CMS-PAS-B2G-17-008.

[33] M. Baak et al. [Gfitter Group], Eur. Phys. J. C 74, 3046 (2014) doi:10.1140/epjc/s10052-014-3046-5 [arXiv:1407.3792 [hep-ph]]. 